Veröffentlichung wird nicht geprüft und es ist nicht ersichtlich, inwieweit tatsächlich Ansprüche an die Verbände übergegangen sind. Auch wenn eine Pauschalisierung grundsätzlich möglich ist, so hat die Möglichkeit der Pauschalisierung ihre Grenzen doch dort, wo ohne Bezug zum Tatsächlichen gehandelt wird.

Insofern müsste auf Grund echter (zufälliger oder sonst repräsentativer) Stichproben der Umfang der relevanten Veröffentlichungen ermittelt und auf den Umfang der demnach an den Verband abgetretenen Ansprüche aller Verbandsmitglieder hochgerechnet werden.
Die EuGH-Entscheidung "Luksan“ ist auch auf diese Problemstellung nicht anwendbar, da hier keine Nutzungsrechte an die Berufsverbände übergehen, sondern nur nicht geltend gemachte Auszahlungsansprüche. [...] (Entscheidung von der Redaktion bearbeitet.)

\title{
Unrechtmäßigkeit einer Anhalteverfügung (§ 8 Abs. 2 Satz 1 KultGüRückG)
} Verwaltungsgericht Köln, Urteil vom 25. April 2012 - 10 K 3537/11

1. Der zeitliche Anwendungsbereich für eine Anhalteverfügung gemäß § 8 Abs. 2 Kult GüRückG ist nur für solche Objekte eröffnet, die nach dem 26. April 2007 aus dem Hoheitsgebiet eines Vertragsstaates verbracht wurden.

2. Voraussetzung für die Rechtmäßigkeit einer Anhalteverfügung ist im Falle der Einleitung eines Bezeichnungsverfahrens gemäß § 6 Abs. 2 Satz 1 KultGüRückG die öffentliche Bekanntmachung desselben vor Erlass der Verfügung. (Leitsätze des Einsenders*)

\section{Tenor}

- Der Bescheid des Beklagten vom 17. Juni 2011 wird aufgehoben.

Der Beklagte trägt die Kosten des Verfahrens.

Das Urteil ist hinsichtlich der Kosten gegen Sicherheitsleistung in Höhe von $110 \%$ des jeweils zu vollstreckenden Betrages vorläufig vollstreckbar.

Die Berufung wird zugelassen.

\section{Tatbestand}

Die Klägerin betreibt unter der aus dem Rubrum ersichtlichen Bezeichnung in Köln ein Auktionshaus, das sich u.a. mit der Versteigerung von Kunstgegenständen, Möbeln und Porzellanen beschäftigt. Die Klägerin gibt für jede ihrer Auktionen vorher einen Katalog heraus, in dem sie die angebotenen Kunstgegenstände bildlich darstellt und beschreibt, einen Schätzpreis angibt und, soweit möglich, die Provenienz nennt.

Für den 18. Juni 2011 kündigte die Klägerin ihre Auktion 984 „Pre-Columbian Art" an. Auch für diese Auktion hatte sie einen Katalog herausgegeben.

Mit Verbalnote vom 17. Juni 2011 ersuchte die Botschaft von Mexiko in Berlin den Beklagten [das Land Nordrhein-Westfalen, Anmerkung der Redaktion] um Anordnung der Anhaltung der im Versteigerungskatalog der Klägerin mit den Katalognummern

Karl-Sax Feddersen, Syndikus des Kunsthauses Lempertz, Repräsentanz Berlin.
199 - 214, 216 - 217, 219 - 223 und 225 sowie 226 versehenen Artefakte. Vorausgegangen war eine Besichtigung der Gegenstände durch den mexikanischen Archäologen Jaime Alejandro Bautista Valdespino am 15. Juni 2011 und eine von ihm am 16. Juni 2011 abgegebene eidesstattliche Versicherung, wonach die Objekte mexikanischen Ursprungs seien. [...]

Mit Bescheid vom 17. Juni 2011 ordnete der Beklagte die Anhaltung der im Versteigerungskatalog der Klägerin mit den Katalognummern 199-214, 216 - 217, 219 - 223 sowie 225 - 226 versehenen Artefakte an. Er ordnete die sofortige Vollziehung der Anhaltung an.

Zur Begründung der Anhaltung führt er im Wesentlichen Folgendes an: Die Anhaltung habe nach $\S 8$ Abs. 2 KultGüRückG zu erfolgen. Es bestehe der dringende Verdacht der unrechtmäßigen Ausführung der Gegenstände aus dem Gebiet der Vereinigten Mexikanischen Staaten. Die Objekte seien mexikanischen Ursprungs. Sie seien von mesoamerikanischen Kulturen aus dem heutigen mexikanischen Staatsgebiet vor der spanischen Eroberung hergestellt worden. Es handle sich demnach um besonders bedeutsames nationales Kulturgut der Vereinigten Mexikanischen Staaten. Die Eintragung in das „öffentliche Verzeichnis für archäologische und historische Denkmäler und Flächendenkmäler" (Registro Publico de Monumentos y Zonas Arqueologicos e Historicos), das im Internet unter der Adresse http://www.registropublico.inah.gob.mx zugänglich sei, werde auf der Grundlage des Gutachtens des Archäologen Jaime Alejandro Bautista Valdespino in Kürze erfolgen. Das förmliche Eintragungsverfahren sei mit der Entsendung des Archäologen eingeleitet worden. Die Artefakte seien unter Verstoß gegen die 
Ausfuhrbestimmungen zum Schutz von Kulturgütern aus dem mexikanischen Staatsgebiet ausgeführt worden. Gemäß Art. 27, 29, 49, 51 des mexikanischen Bundesgesetzes für archäologische, künstlerische und historische Denkmäler und Flächendenkmäler sei die Ausfuhr von beweglichen archäologischen Objekten in Mexiko unabhängig von den Eigentumsverhältnissen untersagt. Der Staat Mexiko habe keine Ausfuhrgenehmigung bezüglich der streitgegenständlichen archäologischen Objekte erteilt. Nach $\S 6$ KultGüRückG sei bei dem Verfahren auf die Vorschriften des antragstellenden Landes, also Mexiko, abzustellen. Die erfolgte Einfuhr der Artefakte ins Bundesgebiet sei ausweislich der Katalognummern nach dem 26. April 2007 erfolgt. Damit sei der Anwendungsbereich des Kulturgüterrückgabegesetzes und des UNESCO-Übereinkommens vom 14. November 1970 eröffnet.

Am 18. Juni 2011 versteigerte die Klägerin die angehaltenen Artefakte unter Vorbehalt. Sie übertrug den Ersteigerern nicht das Eigentum und den Besitz an den Gegenständen.

Am 20. Juni 2011 hat die Klägerin Klage gegen den Bescheid des Beklagten vom 17. Juni erhoben. [...]

\section{Entscheidungsgründe}

Die zulässige Klage ist begründet. Die Anhalteverfügung des Beklagten vom 17. Juni 2011 ist rechtswidrig und verletzt die Klägerin in ihren Rechten ( $§ 113$ Abs. 1 Satz 1 VwGO). Die Beklagte kann die Anhalteverfügung nicht auf $\S 8$ Abs. 2 Satz 1 KultGüRückG stützen.

Danach ordnen die für die Rückgabe des Kulturgutes zuständigen Behörden die Anhaltung von Kulturgut an oder veranlassen die Anordnung durch die dafür zuständige Behörde, wenn sie Kenntnis von Kulturgut erhalten, bei dem der dringende Verdacht besteht, dass es unrechtmäßig aus einem anderen Mitgliedstaat der Europäischen Union oder Vertragsstaat in das Bundesgebiet verbracht worden und an diesen Staat zurückzugeben ist. Die Voraussetzungen der Rückgabepflicht sind in $\S 6$ Abs. 2 KultGüRückG geregelt. [...]

Die Voraussetzungen der Rückgabepflicht sind im vorliegenden Fall nicht gegeben. [...]

Die Klägerin macht zutreffend geltend, dass bereits der zeitliche Anwendungsbereich des Kulturgüterrückgabegesetzes nicht eröffnet ist. Es ist nicht erkennbar, dass es sich bei den angehaltenen Artefakten um solche handelt, die nach dem 26. April 2007 aus dem mexikanischen Hoheitsgebiet in das Bundesgebiet verbracht worden sind. [...] Eine gefestigte Meinung zum zeitlichen Anwendungsbereich des § 6 Abs. 2 Satz 1 KultGüRückG gibt es nicht. Soweit in den wenigen hierzu ergangenen Stellungnahmen (vgl. VG München Beschl. vom 27. Januar 2010 - M 17 E 09.4833 - juris Rdnr. 15; Rietschel Internationale Vorgaben zum Kulturgüterschutz und ihre Umsetzung in Deutschland (2009), Seite 147) vertreten wird, § 6 Abs. 2 Satz
1 KultGüRückG sei dahin auszulegen, dass es für die Eröffnung des zeitlichen Anwendungsbereichs der Vorschrift ausreiche, dass die Gegenstände nach dem 26. April 2007 in das Bundesgebiet verbracht worden seien, ohne dass es auf die Ausfuhr aus dem ersuchenden Vertragsstaat zum vorgenannten Stichtag ankomme, teilt die Kammer diese Auffassung nicht.

Gegen eine solche Auslegung spricht der Wortlaut des § 6 Abs. 2 Satz 1 KultGüRückG. Dieser stellt nicht nur auf eine nach dem 26. April 2007 erfolgte Verbringung der Gegenstände in das Bundesgebiet ab, sondern auf eine nach dem 26. April 2007 aus dem Hoheitsgebiet des Vertragsstaates erfolgte Verbringung der Gegenstände in das Bundesgebiet. Dem Wortlautargument kommt im vorliegenden Fall besonderes Gewicht zu, weil es für den Gesetzgeber ein Leichtes gewesen wäre, die Norm so zu fassen, wie sie der Interpretation durch den Beklagten entspricht. [...]

Auch die Gesetzesbegründung (Vgl. BT-Drs. 16/1371) führt zu keinem von dem zuvor Gesagten abweichenden Verständnis des $\S 6$ Abs. 2 Satz 1 KultGüRückG. [...] So heißt es dort auf Seite 13 linke Spalte (unten): „Weil eine Rückwirkung verfassungsrechtlich äußerst problematisch wäre, beschränken sich die Bestimmungen des Ausführungsgesetzes im Zusammenhang mit dem UNESCO-Kulturgüterabkommen auf zukünftige Sachverhalte nach Inkrafttreten des Übereinkommens für Deutschland. Sie werden deshalb für die Rückgabe von Kulturgut, das in den vergangenen Jahren im Irak gestohlen und illegal ausgeführt worden ist, überwiegend nicht anwendbar sein [Hervorhebung durch das Gericht], aber künftig illegal nach Deutschland verbrachte Kulturgüter erfassen". Dem korrespondiert, dass nach den plausiblen Ausführungen der Klägerin dem Umstand der (mit dem Schutz des deutschen Kunsthandels begründeten) jahrzehntelangen Zurückhaltung der Bundesrepublik beim Beitritt zum UNESCO-Kulturgutübereinkommen im Falle eines Verständnisses der Norm im Sinne des Beklagten nicht hinreichend Rechnung getragen würde.

Unabhängig davon sind die in § 6 Abs. 2 KultGüRückG geregelten Voraussetzungen der Rückgabepflicht auch deshalb nicht gegeben, weil der Vertragsstaat Mexiko zum Zeitpunkt des Erlasses der angegriffenen Anhalteverfügung am 17. Juni 2011 die angehaltenen Kulturobjekte weder als für die Archäologie, Vorgeschichte, Geschichte, Literatur, Kunst und Wissenschaft besonders bedeutsam bezeichnet hatte, noch ein Verfahren zur Bezeichnung eingeleitet und die Einleitung des Verfahrens öffentlich bekannt gemacht hatte.

Der Staat Mexiko hatte die angehaltenen Artefakte zum Zeitpunkt des Erlasses der angefochtenen Anhalteverfügung nicht als besonders bedeutsam bezeichnet. [...]

Der Staat Mexiko hatte allerdings zum Zeitpunkt des Erlasses der angefochtenen Anhalteverfügung ein Verfahren zur Bezeichnung eingeleitet. [...] Der Staat Mexiko hatte die Einleitung des Verfahrens aber zum Zeitpunkt des Erlasses der ange- 
fochtenen Anhalteverfügung nicht öffentlich bekannt gemacht, wie sich aus der Erklärung des Archäologen Valdespino vom 11. Oktober 2011 und aus der Begründung des angegriffenen Bescheides [ „Die Eintragung (...) wird (...) in Kürze erfolgen..”] ergibt. [...]

Dass der Staat Mexiko, wie oben angezeigt, nach Erlass der angegriffenen Anhalteverfügung eine Registrierung der angehaltenen Artefakte im mexikanischen Register für Kulturgüter vorgenommen hat, beeinflusst das Urteil über die Rechtswidrigkeit nicht. [...]

Ein rechtswidrig erlassener Verwaltungsakt wird durch eine spätere Veränderung der Sach- oder Rechtslage aber regelmäßig selbst dann nicht rechtmäßig, wenn es sich bei ihm um einen Verwaltungsakt mit Dauerwirkung handelt. Der Grund hierfür liegt darin, dass es für seine Rechtswidrigkeit konstituierend ist, dass er entweder rechtswidrig erlassen wurde oder jedenfalls eine Verpflichtung zu seiner Rücknahme besteht, [...]. Andernfalls würde die Rechtssicherheit, der der Gesetzgeber bei der Abfassung des Kulturgüterrückgabegesetzes beachtliches Gewicht beigemessen hat, [...], erheblich beeinträchtigt. Die Verwaltung hätte es in der Hand, (ohne Vorliegen der gesetzlichen Voraussetzungen) gewissermaßen „auf Vorrat" eine Anhalteverfügung zu erlassen, um einem Vertragsstaat die Möglichkeit zu eröffnen bzw. die Option offen zu halten, Gegenstände als besonders bedeutsam zu bezeichnen oder ein Verfahren zur Bezeichnung einzuleiten und die Einleitung des Verfahrens öffentlich bekannt zu machen.

Auch würde der vom Gesetzgeber angestrebte faire Interessenausgleich zwischen den Belangen des Kulturgutschutzes auf nationaler wie internationaler Ebene und den Interessen des Kunsthandels, [...], beeinträchtigt. [...]

Es genügt, dass der ersuchende Vertragsstaat bestimmte Gegenstände als besonders bedeutsam bezeichnet oder die Einleitung des Bezeichnungsverfahrens öffentlich bekannt gemacht hat. Diese Sicherungsmechanismen würden entgegen der Vorstellung des Gesetzgebers noch weiter nach vorne verlagert, wenn man eine Eingriffsbefugnis der für die Rückgabe des Kulturgutes zuständigen Behörden bereits dann anerkennen würde, wenn der nach den vorgenannten Vorschriften erforderliche Publizitätsakt noch gar nicht erfolgt ist oder, was hier allerdings nicht der Fall gewesen ist, der ersuchende Vertragsstaat die von ihm beanspruchten Gegenstände noch nicht einmal individualisiert hat.

Der Kulturgutschutz wird durch diese rechtliche Bewertung nicht unzumutbar eingegrenzt. [...] Der Vertragsstaat Mexiko ist auch im konkreten Fall nicht daran gehindert gewesen, den von $\S 6$ Abs. 2 Satz 1 KultGüRückG geforderten Publizitätsakt zeitnah vorzunehmen. Er hatte zumindest seit dem 20. Mai 2011 - vermutlich noch früher - Kenntnis von den von ihm beanspruchten Artefakten.
Unabhängig von dem zuvor Gesagten sind die Voraussetzungen der Rückgabepflicht jedenfalls hinsichtlich der Artefakte mit den Katalognummern 222 (bezüglich der letzten beiden Stücke), 203, 204, 205 (bezüglich des ersten Stücks), 206 (bezüglich des ersten Stücks), 207, 208 (bezüglich des ersten Stücks) 211, 222 und 223 nicht gegeben, weil insoweit die Jahresfrist des $\S 6$ Abs. 2 Satz 1 Nr. 1 KultGüRückG verstrichen ist. Die Gegenstände sind nicht innerhalb eines Jahres, nachdem die zuständige Behörde des betroffenen Vertragsstaates von ihnen Kenntnis erlangen konnte, als besonders bedeutsam bezeichnet worden. [...]

Unabhängig davon ist der Rückgabeanspruch auch hinsichtlich der Artefakte erloschen, die vor 30 Jahren oder früher aus Mexiko herausgebracht worden sind, § 11 Abs. 1 Satz 3 KultGüRückG. Danach erlischt der Rückgabeanspruch spätestens 30 Jahre nach dem Zeitpunkt, in dem das Kulturgut unrechtmäßig aus dem ersuchenden Staat ausgeführt worden ist.

Dem Begehren der Beklagten, den Staat Mexiko bzw. die mexikanische Botschaft nach $\S 65$ VwGO beizuladen, war nicht zu entsprechen. Im - hier vorliegenden - Bereich hoheitlicher Tätigkeit genießen souveräne Staaten nach Völkergewohnheitsrecht, [...], uneingeschränkte Immunität, die auch die für sie handelnden Organe umfasst. Dies schließt die Beiladung zu einem verwaltungsgerichtlichen Verfahren jedenfalls dann aus, wenn der ausländische Staat die Beiladung nicht selbst ausdrücklich beantragt, [...].

Die Kammer hat die Berufung wegen der grundsätzlichen Bedeutung der Rechtssache zugelassen (§ 124a Abs. 1 Satz 1, $\S 124$ Abs. 2 Nr. 3 VwGO). (Entscheidung von dem Einsender bearbeitet.) 